\title{
Condutas de Limitação Terapêutica em Pacientes Internados em Unidade de Terapia Intensiva*
}

\section{Therapeutic Limitation Conducts in Intensive Care Unit Patients}

\author{
Almir Galvão Vieira Bitencourt', Maira Pereira Dantas², Flávia Branco Cerqueira Serra Neves³, \\ Alessandro de Moura Almeida', Rodrigo Morel Vieira de Melo', Ligia Carvalho Albuquerque', \\ Tiana Mascarenhas Godinho ${ }^{1}$, Sydney Agareno ${ }^{4}$, José Mário M. Teles ${ }^{5}$, Augusto M. C. Farias 6 , Otavio H. Messeder
}

\section{RESUMO}

JUSTIFICATIVA E OBJETIVOS: Atualmente, há uma tendência crescente de se buscar o "morrer com dignidade", mais do que prolongar inutilmente o sofrimento de pacientes terminais em unidades de terapia intensiva (UTI). O objetivo deste estudo foi avaliar a utilização de condutas que sugerem limitação terapêutica (LT) em pacientes que foram a óbito em UTI Adulto.

MÉTODO: Trata-se de um estudo exploratório, retrospectivo, que avaliou prontuários médicos de pacientes que foram a óbito na UTI geral de um hospital privado de Salvador-BA, entre janeiro e agosto de 2003, com internação superior a 24 horas na unidade. Os pacientes foram classificados, em relação ao óbito, em "não resposta a medidas de reanimação", "morte encefálica", "decisão de não reanimar" (DNR), "não adoção ou

1. Graduandos da Faculdade de Medicina da Bahia (UFBA).

2. Médica Intensivista, Plantonista da UTI Geral do Hospital Português, Salvador, BA.

3. Graduando da Escola Bahiana de Medicina e Saúde Pública.

4. Médico Intensivista, Coordenador da UTI do Hospital Agenor Paiva, Salvador, BA.

5. Médico Intensivista, Coordenador da UTI do Hospital Salvador e Vice-Coordenador da UTI Geral do Hospital Português, Salvador, BA. 6. Médico intensivista, Coordenador da UTI Geral do Hospital Português, Salvador, BA.

${ }^{*}$ Recebido da Unidade de Terapia Intensiva Geral do Hospital Português, Salvador, BA.

Apresentado em 15 de abril de 2007

Aceito para publicação em 14 de junho de 2007

Endereço para correspondência:

Almir Galvão Vieira Bitencourt

Rua Vanderlei Pinho, 181/802 - Itaigara

41815-270 Salvador, BA

Fone: (71) 8806-0515 / 3451-4063

E-mail: almirgvb@yahoo.com.br

(C)Associação de Medicina Intensiva Brasileira, 2007 retirada de medidas de suporte de vida", sendo estas duas últimas consideradas medidas sugestivas de limitação terapêutica

RESULTADOS: Foram incluídos dados referentes a 67 pacientes, correspondendo a 90,4\% dos pacientes falecidos na unidade durante o período. Destes, $56,7 \%$ eram mulheres e a idade média dos pacientes foi de $66,58 \pm 17,86$ anos. Medidas sugestivas de LT foram encontradas em $59,7 \%$ dos pacientes, sendo a mais importante "não adoção de medidas de suporte" $(35,8 \%)$, seguida de DNR $(17,9 \%)$ e "retirada de medidas de suporte" (6\%). A utilização de fármacos vasoativos e métodos dialíticos foram as medidas de suporte mais omitidas, enquanto antibioticoterapia foi a mais retirada. A utilização de medidas de LT foi mais freqüente nos pacientes clínicos.

CONCLUSÕES: Os dados do presente estudo sugerem altas freqüências de condutas médicas sugestivas de LT em UTI geral no Nordeste do Brasil. Métodos terapêuticos que possam causar desconforto ou sofrimento aos pacientes terminais, como nutrição, sedação e analgesia, raramente foram omitidos ou retirados.

Unitermos: Cuidados Intensivos, Ética Médica, Futilidade Médica, Interrupção do Tratamento, Ordens de não Reanimar.

\section{SUMMARY}

BACKGROUND AND OBJECTIVES: There is a growing tendency of looking for "dying with dignity", rather than to prolong death and suffering of terminal patients on intensive care units (ICU). This study aims to evaluate medical practices that suggest therapeutic limitation $(T L)$ in patients who died in an adult ICU.

METHODS: A retrospective exploratory study was carried out to evaluate medical records of patients who died in a general adult ICU of a private hospital in Sal- 
vador-BA, between January and August of 2003, after at least 24 hours from the admission. The patients were classified, in relation to their deaths, in: "not responding to cardiopulmonary resuscitation", "brain death", "decision not to resuscitate" (DNR) and "withhold or withdrawal life-support measures".

RESULTS: Sixty seven patients were included, corresponding to $90.4 \%$ of the deaths occurred in this ICU during the referred period. The most of them (56.7\%) were women and the patients' mean age was $66.58 \pm$ 17.86 years. Suggestive measures of TL were found in $59.7 \%$ of the patients, being "withhold of life-support measures" the most important (35.8\%), followed by DNR (17.9\%) and "withdrawal of life-support measures" (6\%). The procedures most commonly omitted were use of vasoactive drugs and dialysis, while antibiotics were the most discontinued. The use of TL measures was more frequent in clinical patients.

CONCLUSIONS: The results of the present study suggest high frequencies of medical conducts suggestive of TL in a general ICU in Northeast of Brazil. Therapeutic methods that could cause discomfort or suffering to the patients, as nutrition, sedation and analgesia, were rarely omitted or discontinued.

Key Words: Intensive Care, Medical Ethics, Medical Futility, Withholding Treatment, Resuscitation Orders.

\section{INTRODUÇÃO}

Com o desenvolvimento tecnológico da Medicina, a adoção de medidas de suporte à vida tem permitido que pacientes graves possam ser mantidos por longos períodos nas unidades de terapia intensiva (UTI), dificultando a identificação de limites terapêuticos nesse ambiente. Este prolongamento do processo de morrer, quando não há mais esperança de cura, é conhecido como distanásia, obstinação terapêutica, futilidade médica ou tratamento fútil ${ }^{1}$. Em alguns casos a morte é inevitável, sendo apenas retardada a um alto custo financeiro, social e psicológico para todas as partes envolvidas nesse processo (paciente, familiares e profissionais de saúde) ${ }^{2}$.

Atualmente, há uma tendência crescente de se buscar o "morrer com dignidade", mais do que retardar inutilmente e prolongar o sofrimento do paciente. O médico intensivista passou, então, a se confrontar com decisões de recusar ou suspender tratamentos considerados fúteis ou inúteis ${ }^{3,4}$. A partir desta constatação, vários estudos em todo o mundo têm se preocupado em discutir a freqüência e os fatores que determinam medidas de limitação terapêutica em pacientes internados em UT| ${ }^{5-7}$.

As práticas médicas relacionadas aos cuidados com o fim da vida variam bastante em diferentes países, culturas e regiões ${ }^{8,9}$. Embora este tema já seja amplamente estudado em países desenvolvidos, poucos dados estão disponíveis sobre de que forma essa prática é realizada em países em desenvolvimento.

O objetivo deste estudo foi avaliar a utilização de condutas que sugerem limitação terapêutica em pacientes internados em UTI de um hospital privado no Brasil.

\section{MÉTODO}

Trata-se de um estudo exploratório, retrospectivo, que avaliou prontuários médicos de pacientes internados e que foram a óbito na UTI geral do Hospital Português (HP) em Salvador-BA, Brasil, entre janeiro e agosto de 2003. Foram excluídos os pacientes que permaneceram menos de 24 horas internados na UTI. Esse estudo foi submetido e aprovado pelo Comitê de Ética em Pesquisa do referido hospital.

Trata-se de um hospital privado, com 302 leitos. Possui quatro UTI: coronariana, pós-operatório de cirurgia cardíaca, gastro-hepatologia e geral. A UTI geral, local do estudo, é composta por 24 leitos ocupados por pacientes clínicos e cirúrgicos de diversas especialidades.

Foram colhidas informações referentes às características clínicas e demográficas dos pacientes que incluíram sexo, idade, motivo de internação na UTI, pontuação APACHE II, procedência, tipo de tratamento, comorbidades, tempo de internação e modo de morrer. As seguintes comorbidades foram estudadas: insuficiência cardíaca, hipertensão arterial sistêmica, diabete melito, insuficiência renal crônica, insuficiência respiratória, obesidade, doença de Parkinson, neoplasia, doença de Alzheimer, cirrose hepática, doença pulmonar obstrutiva crônica (DPOC), síndrome da imunodeficiência adquirida (SIDA), quimioterapia, radioterapia, imunossupressão, corticoterapia, insuficiência coronariana, depressão, insuficiência vascular periférica, asma e acidente vascular encefálico. Quanto ao tipo de tratamento os pacientes foram divididos em clínicos e cirúrgicos.

Os pacientes foram classificados quanto aos modos de morrer em: Não resposta a medidas de reanimação, quando houve registro no prontuário de que o paciente recebeu todo o tratamento indicado, incluindo medidas de reanimação cardiopulmonar (RCP) ou tentativas de reanimação; morte encefálica, quando houve regis- 
tro no prontuário de diagnóstico clínico confirmado por exame complementar de acordo com o protocolo utilizado na instituição; decisão de não reanimar, considerado como a não adoção de medidas de RCP em paciente após sofrer parada cardiorrespiratória (PCR), mas que até então tinha recebido todo o tratamento intensivo indicado; não adoção ou retirada de medidas de suporte de vida, quando houve a decisão registrada no prontuário de, respectivamente, não introduzir alguma nova medida terapêutica ou de retirar uma medida previamente instituída que resultou no desenlace natural, excluindo o evento terminal PCR. As medidas de suporte de vida consideradas foram: utilização de fármacos vasopressores, ventilação mecânica, métodos dialíticos, antibioticoterapia, transfusão de sangue e hemoderivados, nutrição, sedação e analgesia. Após essa classificação, os pacientes foram divididos em dois grupos, o primeiro com registro de medidas sugestivas de limitação terapêutica (decisão de não reanimar, não adoção ou retirada de medidas de suporte de vida) e o segundo sem medidas sugestivas de limitação terapêutica (morte encefálica, ou não responsivos às medidas de reanimação). A classificação dos modos de morrer foi realizada por um único observador, obedecendo aos critérios descritos.

Os resultados foram expressos em medidas de tendência central e dispersão, para as variáveis contínuas, e em freqüências simples e relativa, para as variáveis categóricas. Análises univariadas foram realizadas para evidenciar uma possível associação entre a presença de limitação terapêutica e as outras variáveis clínicas e demográficas.

Foi realizada ainda regressão logística para identificação de variáveis associadas com a presença de limitação terapêutica. As seguintes variáveis foram incluídas na análise multivariada: idade, sexo, número de internações prévias em UTI, tempo de permanência em UTI, procedência, tipo de tratamento (clínico e cirúrgico), $\mathrm{APACHE}$, doença de base e comorbidades. $\mathrm{O}$ nível de significância estatística adotado foi de $5 \%$ ( $p=$ 0,05). Para o processamento dos dados foi utilizado o software Statistical Package for Social Science (SPSS) na versão 9.0 .

\section{RESULTADOS}

No período do estudo foram avaliados 707 pacientes internados na UTI, sendo que 13,6\% ( $n=96)$ foram a óbito na unidade. Vinte e dois pacientes faleceram dentro das primeiras 24 horas da internação, sendo excluídos da análise. Foram incluídos os dados de 67 pacientes, correspondendo a 90,4\% dos pacientes falecidos na unidade durante o período avaliado, com mais de 24 horas de internação. A tabela 1 mostra dados clínicos e demográficos dessa população. Os motivos de internação mais comuns foram insuficiência respiratória por infecção pulmonar $(17,9 \%, n=12)$ e choque séptico $(14,9 \%, n=10)$. As comorbidades mais comuns foram neoplasia avançada $(34,3 \%, n=$ 27 ) e doença cardíaca avançada $(10,4 \%, n=7)$.

Tabela 1 - Dados Clínicos e Demográficos da População Estudada ( $\mathrm{n}=67)$.

\begin{tabular}{|c|c|c|c|c|}
\hline & $\begin{array}{c}\text { Todos } \\
(\mathrm{n}=67)\end{array}$ & $\begin{array}{l}\text { Com LT } \\
(n=40)\end{array}$ & $\begin{array}{l}\text { Sem LT } \\
(n=27)\end{array}$ & $\mathrm{p}$ \\
\hline Sexo masculino - $\mathrm{n}(\%)$ & $29(43,3)$ & $17(42,5)$ & $12(44,4)$ & 0,875 \\
\hline Idade (anos) * & $66,58 \pm 17,86$ & $67,75 \pm 16,87$ & $64,85 \pm 19,42$ & 0,650 \\
\hline Idade igual ou superior a 65 anos - n (\%) & $43(64,2)$ & $26(65,0)$ & $17(63,0)$ & 0,865 \\
\hline Tempo de permanência (dias) * & $9,88 \pm 7,97$ & $11,40 \pm 8,49$ & $7,63 \pm 6,65$ & 0,029 \\
\hline Tempo de permanência igual ou superior a 10 dias - $n(\%)$ & $34(50,7)$ & $24(60,0)$ & $10(37,0)$ & 0,065 \\
\hline Neoplasia avançada - n (\%) & $23(34,3)$ & $15(37,5)$ & $8(29,6)$ & 0,506 \\
\hline Valor do APACHE II * & $25,73 \pm 8,15$ & $26,58 \pm 7,82$ & $24,48 \pm 8,60$ & 0,239 \\
\hline Mortalidade estimada em \% * & $51,94 \pm 26,75$ & $53,88 \pm 26,83$ & $49,06 \pm 26,86$ & 0,569 \\
\hline Procedência - n (\%) & & & & 0,258 \\
\hline Emergência & $19(28,4)$ & $12(30,0)$ & $7(25,9)$ & \\
\hline Outras unidades fechadas & $18(26,8)$ & $10(25,0)$ & $8(29,7)$ & \\
\hline Enfermaria & $16(23,9)$ & $12(30,0)$ & $4(14,8)$ & \\
\hline Centro cirúrgico & $10(14,9)$ & $4(10,0)$ & $6(22,2)$ & \\
\hline Outra & $4(6,0)$ & $2(5,0)$ & $2(7,4)$ & \\
\hline Tratamento clínico - n (\%) & $47(70,1)$ & $32(80,0)$ & $15(55,6)$ & 0,032 \\
\hline
\end{tabular}

*Valores expressos em Média \pm DP

LT = Limitação Terapêutica 
Medidas sugestivas de limitação terapêutica foram encontradas em $59,7 \%$ dos pacientes incluídos ( $n=$ 40 ), sendo a mais freqüente delas a não adoção de medidas de suporte. A freqüência de todos os modos de morrer estão descritas na tabela 2. A utilização de fármacos vasopressores e métodos dialíticos foram as medidas de suporte mais omitidas $(65,4$ e $38,5 \%$, respectivamente, dos casos de não adoção de medidas), seguidas de ventilação mecânica em $7,7 \%$ e transfusão de hemoderivados em $3,8 \%$. Nos quatro pacientes em que houve suspensão de medidas terapêuticas, antibioticoterapia foi suspensa em todos (100\%), fármacos vasopressores em dois (50\%) e hemoderivados em um (25\%). Nutrição, analgesia e sedação não foram omitidas ou suspensas em nenhum paciente.

Tabela 2 - Freqüência dos Modos de Morrer $(n=67)$.

\begin{tabular}{lc}
\hline & Freqüência - n (\%) \\
\hline Não responsivos às medidas de reanimação & $25(37,3 \%)$ \\
Não adoção de medidas de suporte & $24(35,8 \%)$ \\
Decisão de não reanimar & $12(17,9 \%)$ \\
Retirada de medidas de suporte & $4(6 \%)$ \\
Morte encefálica & $2(3 \%)$ \\
\hline
\end{tabular}

Em análise univariada não houve diferença estatística entre os dois grupos em relação à idade, sexo, mortalidade estimada pelo APACHE II e presença de neoplasia avançada como comorbidade (Tabela 1). Foi identificada associação estatística entre medidas que sugerem limitação terapêutica e paciente com tratamento clínico, em detrimento daqueles com tratamento cirúrgico $(p=0,032)$, além de maior tempo de permanência na UTI ( $p=0,029)$. Em análise multivariada, apenas o tratamento clínico permaneceu como fator preditor independente de medidas sugestivas de limitação terapêutica (Tabela 3).

Tabela 3 - Fatores Independentes Associados a Medidas Sugestivas de Limitação Terapêutica.

\begin{tabular}{lccc}
\hline & OR & 95\% IC & Significância $(p)$ \\
\hline $\begin{array}{l}\text { Tempo de Internação } \\
\text { maior que 10 dias }\end{array}$ & 2,716 & $0,950-7,771$ & 0,062 \\
Tratamento clínico & 3,399 & $1,104-10,465$ & 0,033 \\
\hline
\end{tabular}

$\mathrm{OR}=$ Odds Ratio

95\% IC = Intervalo de confiança de 95\%

\section{DISCUSSÃO}

Os cuidados com o fim da vida em pacientes críticos vêm sendo amplamente discutidos na literatura, mas, a despeito disso, existe uma grande variedade de con- dutas nos estudos publicados até a presente data. As atitudes dos médicos são diferentes não só em várias regiões do mundo, como também dentro de cada país. Os dados do presente estudo revelam alta prevalência de condutas sugestivas de limitação terapêutica em UTI geral de um hospital privado no Brasil, compatível, contudo, com estudos prévios. Essas condutas foram mais comuns em pacientes clínicos, provavelmente pelo seu perfil crônico, embora não tenha havido relação com idade, doença de base ou mortalidade estimada.

Diversos estudos realizados em países desenvolvidos também buscaram avaliar a freqüência de medidas de limitação terapêutica. Prendergast e col. em estudo multicêntrico realizado nos Estados Unidos com 6.303 pacientes, encontraram uma prevalência dessas medidas em $70 \%$ dos óbitos (excluindo os pacientes com morte encefálica), sendo mais freqüente a retirada de medidas de suporte $(38 \%)^{5}$. Em estudo realizado no Canadá, em três UTI de hospital de ensino, Keenan e col. demonstraram que a não introdução/retirada de medidas de suporte foi a causa de morte mais comum, responsável por $70 \%$ dos óbitos, tendo o prognóstico reservado como principal fator relacionado à tomada de decisão ${ }^{10}$. No estudo ETHICUS ${ }^{6}$, que envolveu 37 UTI de 17 países europeus, foi relatado que $72,6 \%$ dos pacientes que foram a óbito tinham sua terapêutica limitada e que existe uma grande diversidade de condutas entre os diversos países estudados. Nesse estudo, a freqüência média de não adoção de medidas foi $38 \%$, com variação de $16 \%$ a $70 \%$, e a de retirada de medidas foi $33 \%$, variando de $5 \%$ a $69 \%$. Apesar de, na época deste estudo, a eutanásia só ser permitida na Holanda, a presença de medidas de abreviação do processo de morte foi relatada em sete países, sendo responsável por $2 \%$ dos óbitos, variando de 0 a $19 \%$. Os resultados de outros estudos de observação realizados também em países europeus mostraram não adoção ou retirada de medidas em $6 \%$ a $13,5 \%$ dos pacientes internados em UTI e em 35\% a 93\% dos pacientes que foram a óbito na UTI 11-17.

Fora da América do Norte e Europa poucos estudos foram realizados abordando os cuidados com o fim da vida. Yazigi e col. recentemente demonstraram que a não introdução e a retirada de medidas de suporte de vida esteve presente em, respectivamente, $38 \%$ e $7 \%$ dos pacientes que foram a óbito em UTI de hospital universitário no Líbano $^{18}$. Na Índia, Kapadia e col. encontraram uma prevalência total de $34 \%$ de medidas de limitação terapêutica, sendo esta menor no hospi- 
tal público avaliado (19\%) em comparação com três hospitais privados $(40 \% \text { a } 50 \%)^{19}$. Em um centro na África do Sul essa prevalência foi de $86,7 \%{ }^{16}$. Hariharan e col. num estudo realizado numa UTI cirúrgica em Barbados, na América Central, encontraram que 30\% dos pacientes que foram a óbito entre julho de 1999 e junho de 2002 recebiam tratamento agressivo, mesmo após este tratamento ser considerado fútil devido ao prognóstico reservado ${ }^{20}$. No Brasil, Moritz e col. demonstraram que a recusa ou a suspensão de tratamentos foram constatados em $32 \%$ dos óbitos em UTI de um hospital universitário e a futilidade da terapia foi considerada como principal motivo em $100 \%$ destes $\operatorname{casos}^{21}$. Na América do Sul também foram encontrados estudos referentes ao tema em UTI pediátricas, que revelaram uma freqüência de medidas sugestivas de limitação terapêutica em $36 \%$ a $51,9 \%$ dos óbitos nessa população $22-25$.

A análise desses dados permite observar a grande variedade de atitude dos médicos em todo o mundo. Diferenças culturais, econômicas, religiosas, sociais e legais podem justificar as dificuldades encontradas por profissionais, pacientes e familiares ao lidar com esse tipo de decisão. Em estudo recente realizado em seis países europeus e na Austrália, foi relatado que o país de origem era o principal determinante da atitude do médico frente a decisões de limitar ou não suporte terapêutico, embora características individuais dos médicos como idade, crenças religiosas, sexo e experiência prévia com pacientes terminais eram também fortes determinantes ${ }^{26}$. Essas características dificultam o desenvolvimento de consensos internacionais sobre este tema que possam padronizar esse processo de tomada de decisão referente a questões do fim da vida em pacientes críticos. Algumas diretrizes já foram publicadas $^{4,27,28}$, no entanto, ainda não foram totalmente incorporadas à prática clínica e seus princípios, muitas vezes, não têm sido extrapolados para outras populações ${ }^{29}$. Não existem estudos que tenham avaliado diretamente o custo-benefício da retirada/omissão de medidas, nem o tempo entre estas atitudes e o desfecho final, a morte do paciente, de forma que o valor dessa decisão continua sendo subjetivo ${ }^{30}$. Mesmo com amparo legal e religioso, ainda faltam critérios para estabelecer quando é lícito suspender o suporte de vida. Essas questões não são fáceis de serem mensuradas objetivamente e por isso se torna difícil determinar a melhor prática a ser utilizada, devendo cada caso ser individualizado.

Como os próprios pacientes estão geralmente incapa- citados de tomar esta decisão, o papel da família é de grande importância. No entanto, a presença dos familiares nessas discussões também varia, dependendo da região. Na Europa, a opinião dos médicos tem um papel muito mais importante nessas decisões do que nos Estados Unidos, por exemplo, onde a autonomia do paciente é considerada o princípio predominante e os familiares estão mais envolvidos no processo ${ }^{17}$. A presença de outros profissionais de saúde como enfermeiros, fisioterapeutas e psicólogos, religiosos e Comissões de Ética também podem auxiliar. Em nosso meio, os médicos e os familiares foram considerados os elementos que mais freqüentemente participam dessas discussões ${ }^{31}$. Nesse contexto, um importante foco para melhorar a qualidade do atendimento a pacientes terminais na UTI é aprimorar a comunicação entre os médicos, o paciente e a família. Considerando que uma excelência nos cuidados com o fim da vida é importante para a Medicina intensiva de alta qualidade, os médicos deveriam encarar a comunicação com a família com o mesmo cuidado e planejamento que são realizados outros procedimentos na UTI ${ }^{32}$.

Apesar de não haver diferenças éticas, morais e legais entre suspender e não introduzir novas medidas terapêuticas, a segunda atitude é mais freqüente, pois geralmente se prefere omissões a ações ${ }^{17}$. Porém, não deveria haver diferença entre ambas, visto que as duas são consideradas condutas ativas que geralmente resultam na abreviação do processo inevitável de morte do paciente. No presente estudo foi mais freqüente a não adoção de novas medidas de suporte de vida do que a retirada de medidas previamente instituídas. Freqüentemente, no entanto, as duas atitudes são combinadas no mesmo paciente. No estudo ETHICUS, por exemplo, a não adoção de medidas de suporte acompanhou ou precedeu a retirada de outras medidas em $95,4 \%$ dos pacientes que tiveram algum tratamento suspenso ${ }^{6}$.

Apesar de não existir no Brasil a tradição de documentar a ordem de não reanimar como existe em outros países ${ }^{33}$, foi demonstrado neste estudo que a não adoção de medidas de RCP frente ao evento terminal PCR é uma das formas mais comuns de limitação terapêutica. Yaguchi e col. demonstraram, num estudo envolvendo intensivistas de diversos países, que os médicos brasileiros, assim como nos países do sul europeu, preferem dar ordens de não reanimar verbais a escritas ${ }^{34}$. Além disso, em estudo realizado em hospital-escola pediátrico de nível terciário no Brasil, foi constatado que é comum o registro inverídico 
nos prontuários de medidas de RCP em pacientes que não foram reanimados ${ }^{35}$. Devido a estas dificuldades, considerou-se neste estudo como decisão de não reanimar aqueles pacientes que, apesar de já ter recebido todo o tratamento disponível na UTI, não foram submetidos a medidas de RCP. Estudo anterior realizado pelo mesmo grupo que buscou identificar a percepção dos médicos intensivistas sobre a tomada de decisões terapêuticas frente a pacientes terminais, também mostrou que RCP foi o procedimento terapêutico mais omitido nesses casos, seguido de métodos dialíticos, em $87,1 \%$ e $77,4 \%$ dos casos respectivamente ${ }^{31}$. Naquele estudo os métodos dialíticos também foram as medidas de suporte mais retiradas $(80,6 \%)$, seguidas de hemoderivados $(51,7 \%)$, fármacos vasopressores $(38,7 \%)$, antibioticoterapia $(37,1 \%)$ e nutrição parenteral total $(33,9 \%)$. Assim como no estudo atual foi observado ainda que os métodos terapêuticos que podem causar desconforto ou sofrimento aos pacientes terminais, como sedação e analgesia, raramente são omitidos ou retirados. A ventilação mecânica embora algumas vezes seja omitida, raramente é retirada nestas casuísticas, possivelmente por abreviar mais rapidamente o processo de morte dos pacientes do que a retirada de outras medidas.

Como não há uma padronização de condutas no processo de tomada de decisão para os pacientes em estágio terminal, deve-se salientar a importância de documentar estas decisões nos prontuários dos pacientes. Outros estudos já demonstraram as deficiências encontradas na documentação pelos médicos dos cuidados relativos ao fim da vida, inclusive sugerindo a utilização de formulários específicos para esta situação, com o intuito de facilitar o registro destes dados no prontuário ${ }^{36,37}$.

Por se tratar de um estudo retrospectivo, a inferência em relação à conduta tomada depende da qualidade da informação registrada nos prontuários, o que poderia ser evitado em estudos prospectivos. No entanto, apesar das limitações inerentes ao método utilizado, este trabalho é de fundamental importância por descrever uma realidade que vem sendo cada vez mais discutida, mas que ainda é pouco estudada em nosso meio. Os dados do presente estudo ainda podem estar subestimados, visto que este não avaliou pacientes que tiveram sua terapêutica limitada na UTI e que não foram a óbito na unidade, mas sobreviveram ou faleceram após alta da UTI, como relatado em estudos anteriores $^{12,13,15,18}$.

\section{CONCLUSÃO}

Hipócrates, há 2500 anos, dizia que um dos papéis da Medicina é "recusar-se a tratar daqueles que foram vencidos pela doença, entendendo que, diante de tais casos, a medicina torna-se impotente" ${ }^{38}$. Continuar um tratamento considerado fútil é contra todos os princípios fundamentais da ética médica: contra a beneficência, pois não provê nenhum benefício ao paciente; contra a não-maleficência, pois pode causar sofrimento e prejuízo ao paciente; contra a justiça, pois exige custos, tempo e energia que poderiam ser mais bem usadas em outros pacientes; e até mesmo contra a autonomia, pois ninguém quer receber uma terapia fútil que simplesmente prolonga por horas ou dias a morte (inevitável) em estado vegetativo irreversível ${ }^{17}$. A partir desta compreensão, é fácil entender a importância de cada vez mais se incentivar a discussão de temas relacionados aos cuidados com o fim da vida em pacientes internados em UTI. Este trabalho foi um dos primeiros realizados no Brasil que avaliou a freqüência de utilização de medidas de limitação terapêutica em UTI de adultos e demonstrou que a prevalência dessas medidas é elevada nesse hospital.

Entretanto, esta não parece ser uma prática universal no nosso país de dimensões continentais e de heterogêneas condições de assistência e de qualificação profissional. Mesmo porque, medidas de limitação do esforço terapêutico ainda não estão integralmente incorporadas na conduta médica por constrangimentos legais impostos pelo Código Penal Brasileiro, que data de 1940. Felizmente, a Resolução n $1.805 / 2006$ do Conselho Federal de Medicina diminuiu estes constrangimentos, permitindo, aos médicos, a limitação do esforço terapêutico, sem, contudo, deixar de assistir aos pacientes, quanto ao seu conforto físico, emocional e espiritual, como, aliás, foi feito no presente trabalho. Recente publicação sobre cuidados em fim da vida (EOL Care - End of Life Care) em relação à Legislação Brasileira ${ }^{39}$ sugere que, embora a Resolução do Conselho seja normativa, ela não tem força de lei. Esta, só terá força de lei quando o Congresso Nacional aprovar a Reforma do Código Penal. Seguramente, futuros trabalhos, multicêntricos, envolvendo um número e uma heterogeneidade maior de pacientes, poderão ajudar a conhecer melhor a realidade deste quadro no nosso país contribuindo para melhor compreensão das razões para as diferenças nacionais e internacionais relacionadas a este processo de tomada de decisão. 


\section{REFERÊNCIAS}

01. Fisher M - Ethical issues in the intensive care unit. Curr Opin Crit Care, 2004;10:292-298.

02. Moritz RD - Dilemas éticos sobre o fim da vida. RBTI, 2003;15:3-4.

03. Vincent $\mathrm{JL}$ - Forgoing life support in western European intensive care units: the results of an ethical questionnaire. Crit Care Med, 1999;27:1626-1633.

04. Attitudes of the critical care medicine professionals concerning forgoing life-sustaining treatments. The Society of Critical Care Medicine Ethics Committee. Crit Care Med, 1992;20:320-326.

05. Prendergast TJ, Claessens MT, Luce JM - A national survey of end-of-life care for critically ill patients. Am J Respir Crit Care Med, 1998;158:11631167.

06. Sprung CL, Cohen SL, Sjokvist P et al - End-of-life practices in European intensive care units. The Ethicus Study. JAMA, 2003;290:790-797.

07. The SUPPORT principal investigators - A controlled trial to improve care for seriously ill hospitalized patients: the study to understand prognoses and preferences for outcomes and risk of treatments (SUPPORT). JAMA, 1995;274:1591-1598.

08. Pochard F, Abroug F - End-of-life decisions in ICU and cultural specifities. Intensive Care Med, 2005;31:506-507.

09. Vincent $\mathrm{JL}$ - Cultural differences in end-of-life care. Crit Care Med, 2001;29:(Suppl2):N52-N55.

10. Keenan SP, Busche KD, Chen LM et al - A retrospective review of a large cohort of patients undergoing the process of withholding or withdrawal of life support. Crit Care Med, 1997;25:1324-1331.

11. Eidelman LA, Jakobson DJ, Worner TM et al - End-of-life intensive care unit decisions, communication, and documentation: an evaluation of physician training. J Crit Care, 2003;18:11-16.

12. Esteban A, Gordo F, Solsona JF et al - Withdrawing and withholding life support in the intensive care unit: a Spanish prospective multi-centre observational study. Intensive Care Med, 2001;27:1744-1749.

13. Ferrand $E$, Robert R, Ingrand $P$ et al - Withholding and withdrawing of life support in intensive- care units in France: a prospective survey. Lancet, 2001;357:9-14.

14. Gajewska K, Schroeder M, De Marre F et al - Analysis of terminal events in 109 successive deaths in a Belgian intensive care unit. Intensive Care Med, 2004;30:1224-1227.

15. Nolin T, Andersson R - Withdraw of medical treatment in the ICU. A cohort study of 318 cases during 1994-2000. Acta Anaesthesiol Scand, 2003;47:501-507.

16. Turner JS, Michell WL, Morgan CJ et al - Limitation of life support: frequency and practice in a London and a Cape Town intensive care unit. Intensive Care Med, 1996;22:1020-1025.

17. Vincent JL - Ethical principles in end-of-life decisions in different European countries. Swiss Med Wkly, 2004;134:65-68.

18. Yazigi A, Riachi M, Dabbar G - Withholding and withdrawing of life-sustaining treatment in a Lebanese intensive care unit: a prospective observational study. Intensive Care Med, 2005;31:562-567.

19. Kapadia F, Singh M, Divatia J et al - Limitation and withdrawal of intensive therapy at the end of life: practices in intensive care units in Mumbai, India. Crit Care Med, 2005;33:1272-1275.

20. Hariharan S, Moseley HS, Kumar AY et al - Futility-of-care decisions in the treatment of moribund intensive care patients in a developing country. Can J Anaesth, 2003;50:847-852.

21. Moritz RD, Pamplona F - Avaliação da recusa ou suspensão de tratamentos considerados fúteis ou inúteis em UTI. RBTI, 2003;15:40-44.

22. Althabe M, Cardigni G, Vassallo JC et al - Dying in the intensive care unit: collaborative multicenter study about forgoing life-sustaining treatment in Argentine pediatric intensive care units. Pediatr Crit Care Med, 2003;4:164-169.

23. Carvalho PR, Rocha TS, Santo AE et al - Modes of death in PICU of a tertiary hospital. Rev Ass Med Brasil, 2001;47:325-331.

24. Lago PM, Piva J, Kipper D et al - Life support limitation at three pediatric intensive care units in southern Brazil. J Pediatr, 2005;81:111-117.

25. Tonelli HA, Mota JA, Oliveira JS - A profile of the medical conduct preceding child death at a tertiary hospital. J Pediatr, 2005;81:118-125.

26. Miccinesi G, Fischer S, Paci E et al - Physicians' attitudes towards endof-life decisions: a comparison between seven countries. Soc Sci Med, 2005;60:1961-1974.

27. Ethical and moral guidelines for the initiation, continuation, and withdrawal of intensive care. American College of Chest Physicians/Society of Critical Care Medicine Consensus Panel. Chest, 1990;97:949-958.

28. Withholding and withdrawing life-sustaining therapy. This Official Statement of the American Thoracic Society Bioethics Task Force. Am Rev Respir Dis, 1991;144:726-731.

29. Pochard F, Azoulay E, Chevret S et al - French intensivists do not apply American recommendations regarding decisions to forgo life-sustaining therapy. Crit Care Med, 2001;29:1887-1892.

30. Pronovost P, Angus DC - Economics of end-of-life care in intensive care unit. Crit Care Med, 2001;29:(Suppl2):N46-N51.

31. Athanazio RA, Barbetta MC, Bitencourt AGV et al - Decisão de não introduzir ou de retirar tratamentos de suporte para pacientes terminais internados em unidades de terapia intensiva. RBTI, 2005;17:181-184.

32. Curtis JR, Patrick DL, Shannon SE et al - The family conference as a focus to improve communication about end-of-life care in the intensive care unit: opportunities for improvement. Crit Care Med, 2001;29(Suppl2):N26-N33.

33. Weiss GL, Hite CA - The do-not-resuscitate decision: the context, process, and consequences of DNR orders. Death Stud, 2000;24:307-323.

34. Yaguchi A, Truog RD, Curtis JR et al - International differences in endof-life attitudes in the intensive care unit: results of a survey. Arch Intern Med, 2005;165:1970-1975.

35. Torreao LA, Reis AG, Troster EJ et al - Cardiopulmonary resuscitation: discrepancy between the actual cardiopulmonary resuscitation and the documentation in the medical record. J Pediatr, 2000;76:429-433.

36. Kirchhoff KT, Anumandla PR, Foth KT et al - Documentation on withdraw of life support in adult patients in the intensive care unit. Am J Crit Care, 2004;13:328-334.

37. Eidelman LA, Jakobson DJ, Pizov $R$ et al - Foregoing life-sustaining treatment in an Israeli ICU. Intensive Care Med, 1998;24:162-166.

38. Moritz RD - O efeito da informação sobre o comportamento dos profissionais da saúde diante da morte. [tese] Florianópolis: Programa de Pós-Graduação em Engenharia de Produção, Universidade Federal de Santa Catarina, 2002.

39. Soares M, Terzi RG, Piva JP - End-of-life care in Brazil. Intensive Care Med, 2007;33:1014-1017. 